\title{
Der 2,4,6-Tris(trifluormethyl)phenylsubstituent; Beispiele für elektronisch und sterisch stabilisierte niederkoordinierte Hauptgruppenelemente
}

\author{
Mario Scholz, Herbert W. Roesky, Dietmar Stalke, Klaus Keller \\ und Frank T. Edelmann * \\ Institut für Anorganische Chemie der Universität Göttingen, Tammannstraße 4, D-3400 Göttingen (B.R.D.) \\ (Eingegangen den 7. November 1988)
}

\begin{abstract}
An improved synthesis of 1,3,5-tris(trifluoromethyl)benzene (1) is reported. $R_{F} P_{2}$ (3), $R_{F} P_{2}$ (4) and the unusually stable diphosphene $R_{F} P=P R_{F}$ (5) are readily accessible starting from $\left[2,4,6-\left(\mathrm{CF}_{3}\right)_{3} \mathrm{C}_{6} \mathrm{H}_{2}\right] \mathrm{Li}(2)\left(=\mathrm{R}_{\mathrm{F}} \mathrm{Li}\right)$. Treatment of $\mathrm{Me}_{2} \mathrm{~S}(\mathrm{O})=\mathrm{NPCl}_{2}(6)$ with 4 yields the cyclotetraphosphine derivative [ $\mathrm{Me}_{2} \mathrm{~S}(\mathrm{O})=$ $\mathrm{N}]_{2}\left(R_{F}\right)_{2} P_{4}(7)$ which was characterized by an X-ray structure determination (space group: $P 2_{1} / n$ with $a 1569.7(2), b 2293.6(3), c 1823.1(2) \mathrm{pm}, \beta$ 94.94(1) $\left.{ }^{\circ}\right)$. The unsymmetrically substituted diphosphene intermediate $\left[\mathrm{Me}_{2} \mathrm{~S}(\mathrm{O})=\mathbf{N}\right] \mathrm{P}=\mathbf{P R _ { F }}$ can be trapped as an adduct with $\left[\mathrm{Pt}\left(\mathrm{PPh}_{3}\right)_{2}\right]$. The syntheses of $\left(\mathrm{R}_{\mathrm{F}}\right)_{2} \mathrm{AsF}(9)$ and $\left(\mathrm{R}_{\mathrm{F}}\right)_{2} \mathrm{AsH}(10)$ are also described.
\end{abstract}

\section{Zusammenfassung}

Es wird über eine verbesserte Synthese von 1,3,5-Tris(trifluormethyl)benzol (1) berichtet. Ausgehend von $\left[2,4,6-\left(\mathrm{CF}_{3}\right)_{3} \mathrm{C}_{6} \mathrm{H}_{2}\right] \mathrm{Li}$ (2) $\left(=\mathrm{R}_{\mathrm{F}} \mathrm{Li}\right)$ sind $\mathrm{R}_{\mathrm{F}} \mathrm{PCl}_{2}$ (3), $\mathbf{R}_{F} \mathbf{P H}_{2}$ (4) und das ungewöhnlich stabile Diphosphen $R_{F} P=P R_{F}$ (5) leicht zugänglich. Die Umsetzung von $\mathrm{Me}_{2} \mathrm{~S}(\mathrm{O})=\mathrm{NPCl}_{2}$ (6) mit 4 liefert das gemischt substituierte Cyclotetraphosphan $\left[\mathrm{Me}_{2} \mathrm{~S}(\mathrm{O})=\mathrm{N}\right]_{2}\left(\mathbf{R}_{\mathrm{F}}\right)_{2} \mathrm{P}_{4} \quad(7)$, das durch eine Röntgenstrukturanalyse charakterisiert wurde. (Raumgruppe: $P 2_{1} / n$, Gitterkonstanten: $a$ 1569.7(2), b 2293.6(3), c 1823.1(2) pm, $\beta$ 94.94(1) ${ }^{\circ}$ ). Das intermediär auftretende unsymmetrisch substituierte Diphosphen $\left[\mathrm{Me}_{2} \mathrm{~S}(\mathrm{O})=\mathrm{N}\right] \mathrm{P}=\mathrm{PR} \mathbf{R}_{\mathrm{F}}$ läßt sich als Addukt mit $\left[\mathrm{Pt}\left(\mathrm{PPh}_{3}\right)_{2}\right]$ abfangen. Weiterhin wird die Synthese von $\left(\mathrm{R}_{\mathrm{F}}\right)_{2}$ AsF (9) und $\left(\mathrm{R}_{\mathrm{F}}\right)_{2} \mathrm{AsH}(10)$ beschrieben.

Seit der Synthese des ersten stabilen Diphosphens durch Yoshifuji et al. [1] hält das große Interesse an der Chemie niederkoordinierter Phosphor- und Arsenverbindungen unvermindert an. So konnte mittlerweile eine große Anzahl bei Raumtemperatur stabiler Diphosphene, Phosphaarsene und Diarsene isoliert werden [2]. 
In nahezu allen Fällen wurde die Stabilisierung der Doppelbindung durch Einführung sterisch anspruchsvoller Substituenten erreicht. Bewährt haben sich Substituenten wie 2,4,6-(tBu) ${ }_{3} \mathrm{C}_{6} \mathrm{H}_{2}-\left(\right.$ Supermesityl), $\left(\mathrm{Me}_{3} \mathrm{Si}\right)_{2} \mathrm{CH}-,\left(\mathrm{Me}_{3} \mathrm{Si}\right)_{3} \mathrm{C}$ - oder $\mathrm{C}_{5} \mathrm{Me}_{5}$ - In jüngster Zeit konnten insbesondere Weber et al. zeigen, daß auch raumerfüllende Übergangsmetallfragmente wie z.B. $\left(\eta^{5}-\mathrm{C}_{5} \mathrm{Me}_{5}\right)(\mathrm{CO})_{2} \mathrm{Fe}$ in der Lage sind, Diphosphene und Phosphaarsene zu stabilisieren [3]. Über den Einfluß elektronischer Faktoren ist dagegen bislang noch wenig bekannt. Einen ersten Versuch in dieser Richtung machten kürzlich Fscudie et al. [4] mit der Einführung des 2,6-Bis(trifluormethyl)phenyl-Substituenten. Dieser Rest vermag sowohl Stannylene [5] als auch Diphosphene [4] effektiv zu stabilisieren. In der vorliegenden Arbeit soll gezeigt werden, daß sich der leicht zugängliche 2,4,6-Tris(trifluormethyl)phenyl-Substituent vorzüglich für die Synthese niederkoordinierter Phosphor- und Arsen-Verbindungen eignet.

\section{Ergebnisse und Diskussion}

1,3,5-Tris(trifluormethyl)benzol (1) ist durch Fluorierung von Benzol-1,3,5-tricarbonsäure mit $\mathrm{SF}_{4}[6]$ bequem und in größeren Mengen zugänglich. In der Literatur wird die Ausbeute dieser Reaktion mit nur 33\% angegeben [7]. Durch Variation der Reaktionsbedingungen konnten wir die Ausbeute an 1 reproduzierbar auf ca. $90 \%$ steigern:
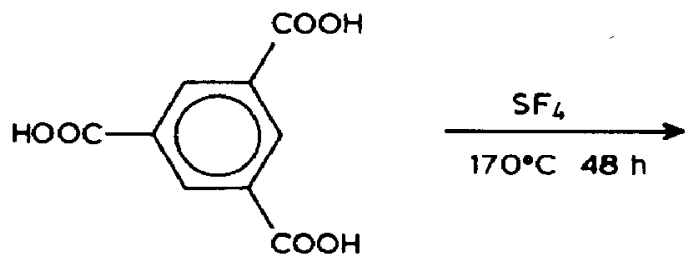

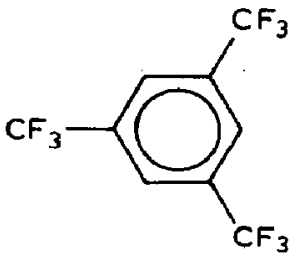

(1)

Die Acidität von 1 ist so hoch, daß sich durch Metallierung mit n-Butyllithium unter milden Bedingungen direkt das Lithiumderivat $\left[2,4,6-\left(\mathrm{CF}_{3}\right)_{3} \mathrm{C}_{6} \mathrm{H}_{2}\right] \mathrm{Li}(2=$ $\mathbf{R}_{\mathrm{F}} \mathrm{Li}$ ) erhalten läßt [7]. Verbindung 2 ist die zentrale Ausgangssubstanz für die nachfolgend beschriebenen Versuche.

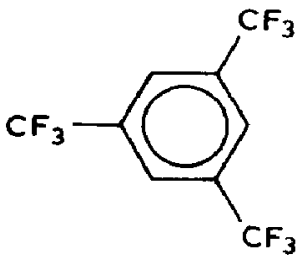

(1)

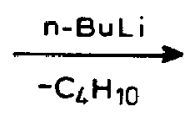

Zur Darstellung des Diphosphens $\mathbf{R}_{F} \mathbf{P}=\mathbf{P R} \mathbf{R}_{\mathbf{F}}$ wurden zunächst die Vorstufen $\mathbf{R}_{F} \mathbf{P C l}_{2}$ (3) und $\mathrm{R}_{\mathrm{F}} \mathrm{PH}_{2}$ (4) synthetisiert. Das Dichlorphosphan 3 ist durch Umsetzung von 2 mit $\mathrm{PCl}_{3}$ in 63\% Ausbeute leicht zugänglich. Auch die Reduktion mit $\mathrm{LiAlH}_{4}$ verläuft problemlos und das primäre Phosphan 4 läßt sich mit $61 \%$ Ausbeute in 
Form einer luftempfindlichen, farblosen Flüssigkeit isolieren:

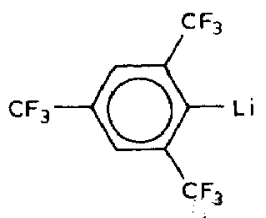

(2)

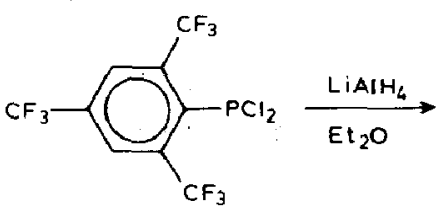

(3)

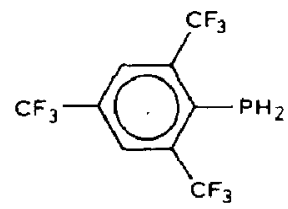

(4)

Der glatte Verlauf dieser Reduktion steht im deutlichen Gegensatz zu Beobachtungen, die am analogen 2,5-Bis(trifluormethyl)phenyl-System gemacht wurden [4]. Hier mißlingt der Versuch, 2,6-( $\left(\mathrm{CF}_{3}\right)_{2} \mathrm{C}_{6} \mathrm{H}_{3} \mathrm{PCl}_{2}$ mit $\mathrm{LiAlH}_{4}$ zu reduzieren, weil $\mathrm{C}-\mathrm{F}$-Bindungen gespalten werden. Die Synthese von 2,6-( $\left(\mathrm{CF}_{3}\right)_{2} \mathrm{C}_{6} \mathrm{H}_{3} \mathrm{PH}_{2}$ war erst durch Anwendung von $\mathbf{n}-\mathrm{Bu}_{3} \mathrm{SnH}$ als Reduktionsmittel erfolgreich. Auch die klassischen Routen zum Diphosphen versagen im Falle der 2,6-Bis(trifluormethyl)phenyl-Derivate. Zur Darstellung von [2,6-( $\left.\left(\mathrm{CF}_{3}\right)_{2} \mathrm{C}_{6} \mathrm{H}_{3} \mathrm{P}\right]_{2}$ mußte ein Umweg über das Germanium-Derivat 2,6-( $\left.\mathrm{CF}_{3}\right)_{2} \mathrm{C}_{6} \mathrm{H}_{3} \mathrm{P}(\mathrm{H}) \mathrm{GeCl}$ beschritten werden [4]. Im Falle der 2,4,6-( $\left(\mathrm{CF}_{3}\right)_{3} \mathrm{C}_{6} \mathrm{H}_{2}-\mathrm{Verbindungen} \mathrm{erhält} \mathrm{man} \mathrm{das} \mathrm{Diphosphen} 5$ leicht durch Kondensation von 3 und 4 in Gegenwart von Diazabicycloundecen (DBU). Über die Darstellung von 5 und verwandten Verbindungen haben wir und Dillon et al. zum ersten Mal berichtet [8].

Das Diphosphen-Derivat 5 fällt als feinkristalliner, blaßgelber Feststoff an (60\% Ausbeute). Es ist weitgehend luftstabil und kann aus siedendem Toluol oder Acetonitril umkristallisiert werden. Die bemerkenswerte kinetische Stabilität des Diphosphens $5 \mathrm{muß}$ hauptsächlich dem elektronischen Einfluß der 2,4,6$\left(\mathrm{CF}_{3}\right)_{3} \mathrm{C}_{6} \mathrm{H}_{2}$-Substituenten zugeschrieben werden. Spektroskopisch und elementaranalytisch läßt sich 5 eindeutig charakterisieren. Im FI-Massenspektrum erscheint bei $m / z 624$ der Molpeak mit 100\% relativer Intensität. Das ${ }^{31} \mathrm{P}$-NMR-Spektrum zeigt bei $\delta 474 \mathrm{ppm}$ elf Peaks eines Multipletts, das vereinfacht als $\mathrm{X}_{2} \mathrm{~A}_{12}$-Spektrum gedeutet werden kann. Die extreme Tieffeldverschiebung beweist das Vorliegen

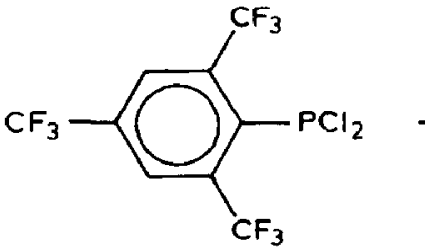

(3)

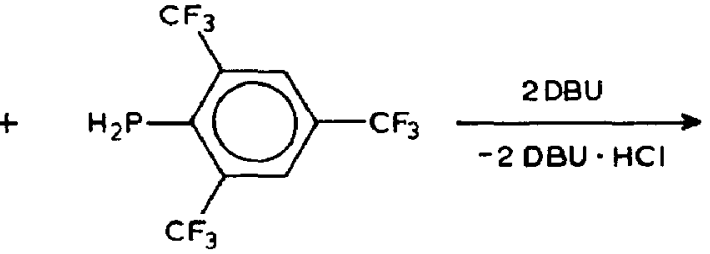

(4)

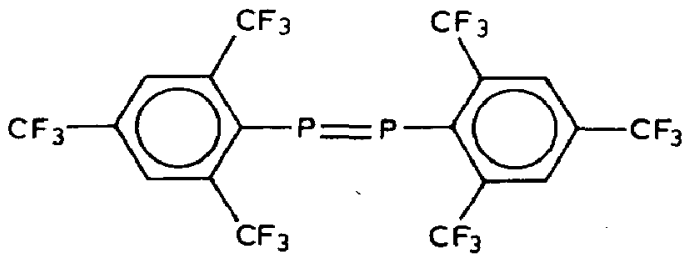


einer Diphosphen-Bindung. Die Reaktivität von 5 scheint begrenzt. So bildet 5 beispielsweise kein Addukt mit Vanadocen und läßt sich mit $\mathrm{HBF}_{4}$ nicht protonieren.

Nachdem gezeigt werden konnte, daß der 2,4,6-Tris(trifluormethyl)phenyl-Substituent die $-\mathrm{P}=\mathrm{P}$-Doppelbindung ungewöhnlich gut $\mathrm{zu}$ stabilisieren vermag, sollte nun der Einfluß eines Substituenten mit gegenlaufigem elektronischen Effekt untersucht werden. Frühere Arbeiten hatten zum Ergebnis, daß Übergangsmetalle in hohen formalen Oxidationsstufen durch Substituenten wie $\mathbf{P h}_{3} \mathrm{P}=\mathbf{N}-[9,10]$, $\left(\mathrm{CF}_{3}\right)_{2} \mathrm{C}=\mathrm{N}$ - [11] oder $\mathrm{Me}_{2} \mathrm{~S}(\mathrm{O})=\mathrm{N}$ - [12] stabilisiert werden. Es war daher von Interesse, einen dieser Reste mit dem 2,4,6-( $\left(\mathrm{CF}_{3}\right)_{3} \mathrm{C}_{6} \mathrm{H}_{2}$-Substituenten am Diphosphen-System zu kombinieren. Dazu wurde zunächst das noch nicht beschriebene Dichlor $(S, S$-dimethylsulfoximino)phosphan 6 synthetisiert.

$\mathrm{Me}_{2} \mathrm{~S}(\mathrm{O})=\mathrm{NSiMe}_{3}+\mathrm{PCl}_{3} \frac{\mathrm{Et}_{2} \mathrm{O}}{-\mathrm{Me}_{3} \mathrm{SiCl}} \mathrm{Me}_{2} \mathrm{~S}(\mathrm{O})=\mathrm{NPCl}_{2}$

Die Reaktion von $S, S$-Dimethyl- $N$-trimethylsilylsulfoximin mit überschüssigem $\mathrm{PCl}_{3}$ verläuft bei $-70^{\circ} \mathrm{C}$ rasch und quantitativ und liefert 6 mit $93 \%$ Ausbeute in Form eines niedrigschmelzenden, farblosen Feststoffs. Eine nachfolgend durchgeführte Umsetzung von 6 mit 3 in Gegenwart von DBU führte nicht zum gewünschten unsymmetrisch substituierten Diphosphen $\left(\mathrm{CF}_{3}\right)_{3} \mathrm{C}_{6} \mathrm{H}_{2} \mathrm{P}=\mathrm{PN}=(\mathrm{O}) \mathrm{SMe}_{2}$ [13]. Stattdessen konnte in geringer Ausbeute das Cyclotetraphosphan 7 isoliert werden.

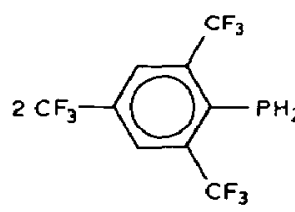

(4)

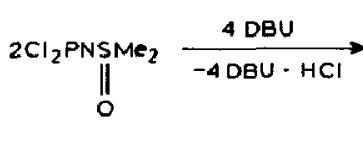

(6)

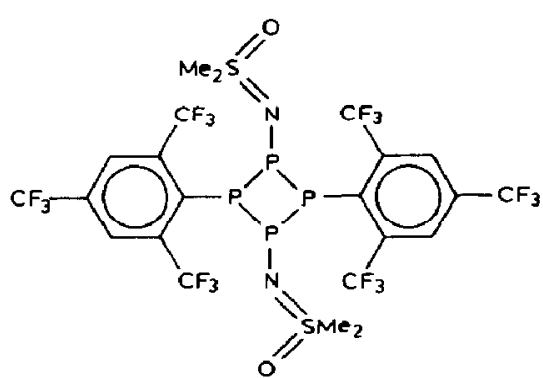

(7)

Offenbar ist die Kombination eines $+M$-Substituenten mit dem elektronenziehenden $\left(\mathrm{CF}_{3}\right)_{3} \mathrm{C}_{6} \mathrm{H}_{2}$-Rest nicht geeignet, ein bei Raumtemperatur isolierbares Diphosphen zu stabilisieren. Das kristalline, gelbe Cyclotetraphosphan wurde durch eine Einkristall-Röntgenstrukturanalyse charakterisiert. Eine bei der Reaktion zwischenzeitlich auftretende Rotfärbung der Lösung ließ auf das intermediäre Auftreten von $\mathbf{R}_{\mathrm{F}} \mathrm{P}=\mathrm{PN}=(\mathrm{O}) \mathrm{SMe}_{2}$ schließen. Wenngleich auch eine Isolierung dieses Diphosphens nicht gelang, konnte es doch als Addukt mit dem carbenanalogen Fragment $\left[\mathrm{Pt}\left(\mathrm{PPh}_{3}\right)_{2}\right]$ abgefangen werden. Dazu wurde die Reaktion von $\mathbf{R}_{\mathrm{F}} \mathrm{PH}_{2}$ mit $\mathrm{Me}_{2} \mathrm{~S}-$ (O) $=\mathrm{NPCl}_{2}$ bei $-78^{\circ} \mathrm{C}$ durchgeführt und das rote $\mathrm{Zwischenprodukt} \mathrm{bei}-60^{\circ} \mathrm{C}$ mit $\left(\mathrm{Ph}_{3} \mathrm{P}\right)_{2} \mathrm{PtC}_{2} \mathrm{H}_{4}$ zur Reaktion gebracht. Dabei konnte orangefarbenes, kristallines $\left.\left(\mathrm{CF}_{3}\right)_{3} \mathrm{C}_{6} \mathrm{H}_{2} \stackrel{\mathrm{PP}\left[\mathrm{N}=(\mathrm{O}) \mathrm{SMe}_{2}\right] \mathrm{Pt}}{\mathrm{PPPh}}\right)_{2}(8)$ mit 44\% Ausbeute isoliert werden. Auch Arsen-Derivate des 2,4,6-Tris(trifluormethyl)phenyl-Substituenten sind problemlos zugänglich. So entsteht bei der Reaktion von 2 mit $\mathrm{AsF}_{3}$ in guter Ausbeute das Disubstitutionsprodukt $\left(R_{F}\right)_{2} A s F(9)$, das nach Sublimation in Form von farblosen Kristallen anfällt. Die Reduktion von 9 mit $\mathrm{LiAlH}_{4}$ liefert das ebenfalls sublimierbare sekundäre Arsan $\left(R_{F}\right)_{2} A s H(10)$. 


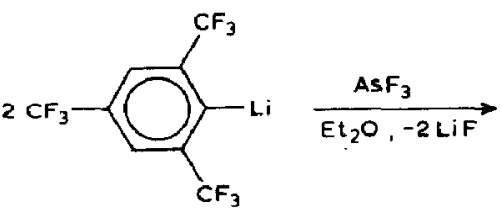

(2)

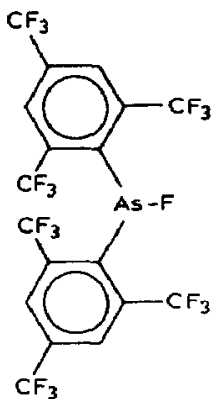

(9)

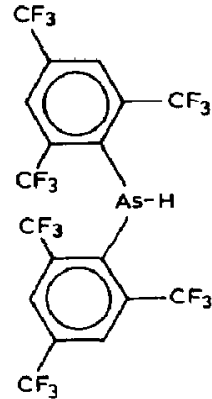

(10)

Insgesamt zeigen die hier vorgestellten Beispiele, daß sich der $2,4,6-\left(\mathrm{CF}_{3}\right)_{3} \mathrm{C}_{6} \mathrm{H}_{2}-$ Substituent sehr gut für die Synthese niederkoordinierter Phosphor- und Arsenverbindungen eignet.

\section{Röntgenstrukturbestimmung von 7}

Verbindung 7 kristallisiert als $\mathbf{P}_{4}$-Ring mit zwei unabhängigen Molekülen in der asymmetrischen Einheit. Atomkoordinaten und isotrope Thermalparameter sind in Tab. 1 aufgeführt. Die $\left(\mathrm{CF}_{3}\right)_{3} \mathrm{C}_{6} \mathrm{H}_{2^{-}}$und die $\mathrm{Me}_{2} \mathrm{~S}(\mathrm{O})=\mathrm{N}-\mathrm{Substituenten}$ befinden sich jeweils auf der gleichen Seite relativ zum $P_{4}$-Ringschwerpunkt (Fig. 1). Die Gruppen zeigen jedoch Unterschiede: Je eine $\left(\mathrm{CF}_{3}\right)_{3} \mathrm{C}_{6} \mathrm{H}_{2}$-Gruppe steht mit zwei $\mathrm{CF}_{3}$-Gruppen ekliptisch zu den beiden $\mathrm{Me}_{2} \mathrm{~S}(\mathrm{O})=\mathrm{N}-\mathrm{Substituenten}$, die andere dagegen gestaffelt. Eine $\mathrm{Me}_{2} \mathrm{~S}(\mathrm{O})=\mathrm{N}$-Einheit weist über den Schwerpunkt des Rings, die andere von ihm weg.

Diese räumliche Anordnung hat interessante Auswirkungen auf das $\mathrm{P}_{4}$-Ringgerüst. Die Ringbindungen der Phosphoratome, die die ekliptisch stehenden $\left(\mathrm{CF}_{3}\right)_{3} \mathrm{C}_{6} \mathrm{H}_{2^{-}}$ Einheiten tragen (P4 und P6), sind im Mittel um $4.5 \mathrm{pm}$ länger als die der gegenüberliegenden Phosphoratome (P3 und P8). Die kurze P-P-Bindung beträgt im Mittel $222.2 \mathrm{pm}$ und liegt damit im Bereich der für andere vergleichbare Systeme gefundenen Bindungslängen [14,15]. Die mittlere lange P-P-Bindung beträgt 226.9 pm (Tab. 2). Die $\mathrm{P}-\mathrm{N}-\mathrm{S}-\mathrm{Bindungswinkel} \mathrm{(Tab.} \mathrm{3)} \mathrm{der} \mathrm{Me}_{2} \mathrm{~S}(\mathrm{O})=\mathrm{N}$-Gruppen, die über den Ring ragen, sind gegenüber denen der abgewandten Substituenten um $10^{\circ}$ aufgeweitet. Dort ist der sterische Druck des Rings besonders groß. Bemerkenswert erscheinen noch die $\mathrm{P}_{4}$-Ring-Faltungswinkel. Sie liegen im Bereich vergleichbarer Systeme [13-18], unterscheiden sich jedoch für beide Moleküle um $8^{\circ}$. Im Molekül

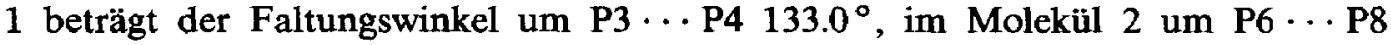
$140.8^{\circ}$. Diese Differenz kann als Argument dafür gesehen werden, daß das Faltungspotential eines $\mathrm{P}_{4}$-Rings sehr klein ist. In diesen beiden chemisch gleich substituierten Cyclotetraphosphan-Molekülen wird der Faltungswinkel von Packungseffekten im Kristallgitter beeinflußt.

Weitere Einzelheiten zur Kristallstrukturuntersuchung können beim Fachinformationszentrum Energie, Physik, Mathematik GmbH, D-7514 EggensteinLeopoldshafen 2, unter Angabe der Hinterlegungsnummer CSD-53415, der Autoren und des Zeitschriftenzitats angefordert werden.

\section{Experimenteller Teil}

Mit Ausnahme der Darstellung von 1 wurden alle Reaktionen unter $\mathbf{N}_{2}$ in sorgfältig getrockneten Lösungsmitteln durchgeführt. ${ }^{1} \mathrm{H}-,{ }^{19}$ F-NMR-Spektren: 
Tabelle 1

Atomkoordinaten $\left(\times 10^{4}\right)$ und äquivalente isotrope Thermalparameter $\left(\mathrm{pm}^{2} \times 10^{-1}\right)$

\begin{tabular}{|c|c|c|c|c|}
\hline Atom & $x$ & $y$ & $z$ & $U(\mathrm{eq})$ \\
\hline $\mathrm{P}(1)$ & 6584(1) & $-715(1)$ & $10029(1)$ & $24(1)$ \\
\hline $\mathbf{P}(2)$ & $7967(1)$ & $-200(1)$ & $9088(1)$ & $21(1)$ \\
\hline $\mathbf{P}(3)$ & $7331(1)$ & $114(1)$ & $10057(1)$ & $23(1)$ \\
\hline $\mathbf{P}(4)$ & $7818(1)$ & $-1072(1)$ & $9639(1)$ & $24(1)$ \\
\hline$S(1)$ & $7263(1)$ & $-859(1)$ & $11558(1)$ & $30(1)$ \\
\hline $\mathbf{S}(2)$ & $9515(1)$ & $329(1)$ & $8797(1)$ & $26(1)$ \\
\hline$O(1)$ & $8139(3)$ & $-980(3)$ & $11428(3)$ & $43(2)$ \\
\hline$O(2)$ & $9095(3)$ & $518(3)$ & $8103(3)$ & $37(2)$ \\
\hline $\mathbf{N}(1)$ & $9011(3)$ & $-18(3)$ & $9335(3)$ & $25(2)$ \\
\hline $\mathbf{N}(2)$ & $6562(3)$ & $-897(3)$ & $10927(3)$ & $27(2)$ \\
\hline $\mathbf{F}(1)$ & $6105(2)$ & $-123(2)$ & 8575(2) & $37(2)$ \\
\hline$F(2)$ & $5023(2)$ & $-40(2)$ & $9212(2)$ & $35(1)$ \\
\hline$F(3)$ & $5063(3)$ & $424(2)$ & $8198(2)$ & $45(2)$ \\
\hline $\mathbf{F}(4)$ & $4597(3)$ & $2338(2)$ & $8855(3)$ & $55(2)$ \\
\hline$F(5)$ & $5455(3)$ & $2780(2)$ & $9664(3)$ & $59(2)$ \\
\hline$F(6)$ & $5824(3)$ & $2646(3)$ & $8566(3)$ & $69(2)$ \\
\hline$F(7)$ & $7648(3)$ & $1101(2)$ & $11194(2)$ & $53(2)$ \\
\hline$F(8)$ & $7922(3)$ & $1915(2)$ & $10714(3)$ & $54(2)$ \\
\hline $\mathbf{F}(9)$ & $8453(3)$ & $1137(2)$ & $10299(3)$ & $48(2)$ \\
\hline$F(10)$ & $8726(3)$ & $-822(2)$ & $7795(2)$ & $40(2)$ \\
\hline$F(11)$ & $9202(3)$ & $-1687(2)$ & $7712(2)$ & $44(2)$ \\
\hline$F(12)$ & $9214(2)$ & $-1277(2)$ & $8763(2)$ & $38(2)$ \\
\hline$F(13)$ & $6980(3)$ & $-2718(2)$ & $6490(2)$ & $57(2)$ \\
\hline$F(14)$ & $5940(3)$ & $-2115(3)$ & $6449(3)$ & $78(2)$ \\
\hline$F(15)$ & $5856(3)$ & $-2918(3)$ & $7029(3)$ & $74(2)$ \\
\hline$F(16)$ & $5599(3)$ & $-1829(2)$ & $9619(2)$ & $46(2)$ \\
\hline$F(17)$ & $6832(3)$ & $-2031(2)$ & $10167(2)$ & $39(2)$ \\
\hline $\mathbf{F}(18)$ & $6120(3)$ & $-2681(2)$ & $9530(3)$ & $50(2)$ \\
\hline $\mathrm{C}(1)$ & $6683(4)$ & $755(3)$ & $9709(3)$ & $23(2)$ \\
\hline$C(2)$ & $5940(4)$ & $785(4)$ & $9202(4)$ & $30(3)$ \\
\hline$C(3)$ & $5536(4)$ & $1307(4)$ & $9033(4)$ & $32(3)$ \\
\hline$C(4)$ & $5839(5)$ & $1822(4)$ & $9325(4)$ & $36(3)$ \\
\hline$C(5)$ & $6564(5)$ & $1823(3)$ & $9815(4)$ & $31(3)$ \\
\hline$C(6)$ & 6976(4) & $1298(3)$ & $10002(4)$ & $27(2)$ \\
\hline$C(7)$ & $5540(4)$ & $258(3)$ & $8808(3)$ & $30(3)$ \\
\hline $\mathrm{C}(8)$ & $5430(4)$ & $2399(4)$ & $9108(4)$ & $53(4)$ \\
\hline$C(9)$ & $7745(5)$ & $1360(3)$ & $10549(4)$ & $38(3)$ \\
\hline$C(10)$ & $7437(4)$ & $-1556(3)$ & $8849(3)$ & $26(2)$ \\
\hline$C(11)$ & $7888(4)$ & $-1589(3)$ & 8191(3) & $23(2)$ \\
\hline$C(12)$ & $7532(5)$ & $-1886(3)$ & $7574(4)$ & $27(2)$ \\
\hline$C(13)$ & $6779(5)$ & $-2199(4)$ & $7585(4)$ & $38(3)$ \\
\hline$C(14)$ & $6419(4)$ & $2251(4)$ & $8243(4)$ & $37(3)$ \\
\hline$C(15)$ & $6744(4)$ & $-1956(3)$ & $8859(4)$ & $28(2)$ \\
\hline$c(16)$ & $8742(4)$ & $-1340(3)$ & $8120(3)$ & $29(3)$ \\
\hline $\mathrm{C}(17)$ & 6394(4) & $-2484(3)$ & 6892(4) & $47(3)$ \\
\hline$C(18)$ & $6324(4)$ & $-2110(3)$ & $9543(4)$ & $36(3)$ \\
\hline$C(19)$ & $6942(6)$ & $-1345(4)$ & $12216(4)$ & $55(4)$ \\
\hline$C(20)$ & $7216(5)$ & $-179(4)$ & $12016(4)$ & $40(3)$ \\
\hline $\mathrm{C}(21)$ & $10435(4)$ & $-78(4)$ & $8632(4)$ & $38(3)$ \\
\hline$C(22)$ & $9969(5)$ & $940(4)$ & $9269(4)$ & $42(3)$ \\
\hline $\mathbf{P}(5)$ & $6560(1)$ & $812(1)$ & $5156(1)$ & $23(1)$ \\
\hline $\mathbf{P}(6)$ & $7776(1)$ & $1149(1)$ & $4703(1)$ & $22(1)$ \\
\hline $\mathbf{P}(7)$ & $7874(1)$ & $280(1)$ & $4125(1)$ & $22(1)$ \\
\hline
\end{tabular}


Tabelle 1 (Fortsetzung)

\begin{tabular}{|c|c|c|c|c|}
\hline Atom & $x$ & $y$ & $z$ & $U($ eq $)$ \\
\hline $\mathbf{P}(8)$ & $7179(1)$ & $-52(1)$ & $5041(1)$ & $23(1)$ \\
\hline$O(3)$ & $8287(3)$ & $899(3)$ & $6415(3)$ & $45(2)$ \\
\hline$O(4)$ & $8932(3)$ & $-487(3)$ & $3145(3)$ & $52(2)$ \\
\hline$S(3)$ & $7435(1)$ & $813(1)$ & $6626(1)$ & $27(1)$ \\
\hline$S(4)$ & $9382(1)$ & $-295(1)$ & $3823(1)$ & $29(1)$ \\
\hline$N(3)$ & $6676(3)$ & $938(3)$ & $6073(3)$ & $28(2)$ \\
\hline$N(4)$ & $8891(3)$ & $59(3)$ & $4374(3)$ & $28(2)$ \\
\hline$F(19)$ & $6004(2)$ & $464(2)$ & $3647(2)$ & $38(2)$ \\
\hline$F(20)$ & $4930(2)$ & $309(2)$ & $4288(2)$ & $39(2)$ \\
\hline$F(21)$ & $4880(3)$ & $49(3)$ & $3158(2)$ & $56(2)$ \\
\hline$F(22)$ & $4472(5)$ & $-2234(4)$ & $3665(4)$ & $169(5)$ \\
\hline$F(23)$ & $5623(4)$ & $-2411(3)$ & $3195(5)$ & $157(5)$ \\
\hline$F(24)$ & $4770(7)$ & $-1862(4)$ & $2732(5)$ & $182(6)$ \\
\hline$F(25)$ & $7234(3)$ & $-1164(2)$ & $5975(2)$ & $57(2)$ \\
\hline$F(26)$ & $8139(3)$ & $-1153(2)$ & $5147(3)$ & $57(2)$ \\
\hline$F(27)$ & $7452(3)$ & $-1934(2)$ & $5365(3)$ & $66(2)$ \\
\hline$F(28)$ & $6769(3)$ & $2094(2)$ & $5259(2)$ & $39(2)$ \\
\hline$F(29)$ & $6067(3)$ & $2756(2)$ & $4635(2)$ & $49(2)$ \\
\hline$F(30)$ & $5524(2)$ & 1913(2) & 4711(2) & $42(2)$ \\
\hline$F(31)$ & $6868(3)$ & 2793(3) & $1576(3)$ & $73(2)$ \\
\hline$F(32)$ & $5747(4)$ & $2983(3)$ & $2114(3)$ & $96(3)$ \\
\hline$F(33)$ & $5848(4)$ & $2186(3)$ & $1540(3)$ & $98(3)$ \\
\hline$F(34)$ & $9084(3)$ & $1730(2)$ & $2735(2)$ & $46(2)$ \\
\hline$F(35)$ & $8576(3)$ & $870(2)$ & $2804(2)$ & $41(2)$ \\
\hline$F(36)$ & $9121(2)$ & $1308(2)$ & $3777(2)$ & $39(2)$ \\
\hline$C(23)$ & $7368(4)$ & $1642(3)$ & $3927(3)$ & $24(2)$ \\
\hline$C(24)$ & $6669(4)$ & $2027(3)$ & $3948(4)$ & $31(3)$ \\
\hline$C(25)$ & $6343(5)$ & $2330(3)$ & $3330(4)$ & $33(3)$ \\
\hline$C(26)$ & $6689(5)$ & $2274(3)$ & $2668(4)$ & $32(3)$ \\
\hline$C(27)$ & $7430(5)$ & $1957(3)$ & $2650(4)$ & $32(3)$ \\
\hline$C(28)$ & $7776(4)$ & $1653(3)$ & $3250(4)$ & $29(2)$ \\
\hline$C(29)$ & $6255(4)$ & $2186(3)$ & $4640(4)$ & $35(3)$ \\
\hline$C(30)$ & $6296(5)$ & $2560(3)$ & 1992(4) & $52(4)$ \\
\hline$C(31)$ & $8638(4)$ & $1385(3)$ & $3148(3)$ & $27(3)$ \\
\hline$C(32)$ & $6455(4)$ & $603(3)$ & $4560(3)$ & $24(2)$ \\
\hline$C(33)$ & $5742(4)$ & $-515(4)$ & $4009(4)$ & $33(3)$ \\
\hline$C(34)$ & $5315(5)$ & $-980(4)$ & $3665(4)$ & $35(3)$ \\
\hline$C(35)$ & $5559(5)$ & $-1534(4)$ & $3834(5)$ & $43(3)$ \\
\hline$C(36)$ & $6236(5)$ & $-1646(4)$ & $4382(5)$ & $45(3)$ \\
\hline$C(37)$ & $6656(5)$ & $-1196(3)$ & 4731(4) & $31(3)$ \\
\hline$C(38)$ & $5398(4)$ & $82(3)$ & $3782(3)$ & $31(3)$ \\
\hline$C(39)$ & $5124(5)$ & $-2014(4)$ & $3407(5)$ & $80(5)$ \\
\hline$C(40)$ & $7372(5)$ & $-1350(3)$ & $5304(4)$ & $42(3)$ \\
\hline$C(41)$ & $7376(5)$ & $110(4)$ & $6989(4)$ & $44(3)$ \\
\hline$C(42)$ & $7270(6)$ & $1269(5)$ & $7368(4)$ & $59(4)$ \\
\hline$C(43)$ & $9841(5)$ & $-900(4)$ & $4287(4)$ & $42(3)$ \\
\hline$C(44)$ & $10297(5)$ & $109(4)$ & $3624(5)$ & 43(3) \\
\hline
\end{tabular}

a Äquivalente isotrope $U$ berechnet als ein Drittel der Spur des orthogonalen $U_{i j}$ Tensors.

Bruker WP 80 SY (80.13 MHz, 75.39 MHz). ${ }^{31}$ P-NMR-Spektren: Bruker AM 250 (101.26 MHz). Massenspektren: CH-5 Varian MAT. IR-Spektren: Perkin-Elmer Spektrograph 735 B. Elementaranalysen: Mikroanalytisches Labor Beller, Göttingen 


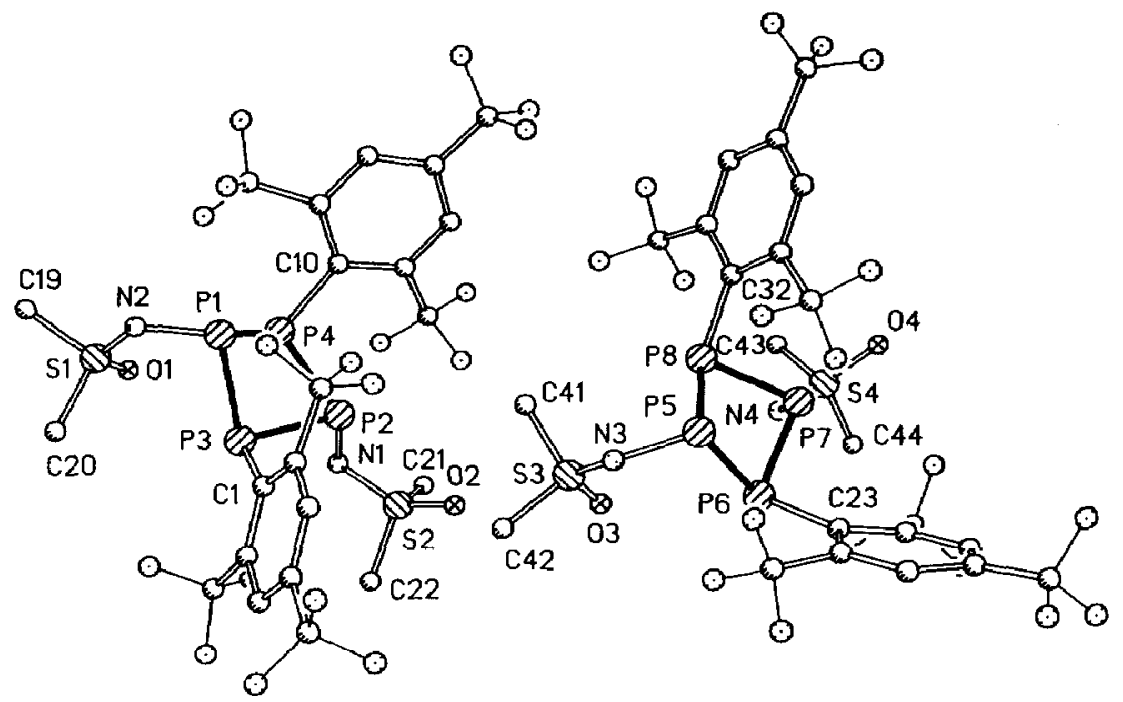

Fig. 1. Die Struktur von 7 im Kristall.

Tabelle 2

Ausgewählte Bindungsabstände (pm)

\begin{tabular}{|c|c|c|c|}
\hline$P(1)-P(3)$ & $223.2(3)$ & $\mathbf{P}(\mathbf{1})-\mathbf{P}(\mathbf{4})$ & $227.4(3)$ \\
\hline$P(1)-N(2)$ & $169.3(6)$ & $\mathbf{P}(2)-\mathbf{P}(3)$ & $222.2(3)$ \\
\hline$P(2)-P(4)$ & $225.9(3)$ & $P(2)-N(1)$ & $171.2(5)$ \\
\hline$P(3)-C(1)$ & $186.7(7)$ & $P(4)-C(10)$ & $187.5(7)$ \\
\hline$S(1)-O(1)$ & $144.3(5)$ & $S(1)-N(2)$ & $152.4(5)$ \\
\hline$S(1)-C(19)$ & $174.4(9)$ & $S(1)-C(20)$ & $177.4(8)$ \\
\hline$S(2)-O(2)$ & $144.3(5)$ & $S(2)-N(1)$ & $153.5(6)$ \\
\hline$S(2)-C(21)$ & $176.6(8)$ & $S(2)-C(22)$ & $176.2(8)$ \\
\hline$C(1)-C(2)$ & $142.6(9)$ & $C(1)-C(6)$ & $141.6(10)$ \\
\hline$C(2)-C(3)$ & $137.8(12)$ & $C(2)-C(7)$ & $151.5(10)$ \\
\hline$C(3)-C(4)$ & $136.4(12)$ & $C(4)-C(5)$ & $138.5(10)$ \\
\hline$C(4)-C(8)$ & $151.0(12)$ & $C(5)-C(6)$ & $139.3(11)$ \\
\hline$C(6)-C(9)$ & $150.4(9)$ & $\mathrm{C}(10)-\mathrm{C}(11)$ & $144.6(9)$ \\
\hline$C(10)-C(15)$ & $142.4(10)$ & $C(11)-C(12)$ & $139.0(9)$ \\
\hline$C(11)-C(16)$ & $147.3(9)$ & $\mathrm{C}(12)-\mathrm{C}(13)$ & $138.4(11)$ \\
\hline$C(13)-C(14)$ & $137.4(11)$ & $C(13)-C(17)$ & $150.3(10)$ \\
\hline$C(14)-C(15)$ & $137.2(10)$ & $C(15)-C(18)$ & $150.2(10)$ \\
\hline$P(5)-P(6)$ & $227.9(3)$ & $P(5)-P(8)$ & $222.5(3)$ \\
\hline$P(5)-N(3)$ & $169.2(6)$ & $P(6)-P(7)$ & $226.6(3)$ \\
\hline$P(6)-C(23)$ & $188.0(7)$ & $\mathbf{P}(7)-\mathbf{P}(8)$ & $220.9(3)$ \\
\hline$P(7)-N(4)$ & $169.9(6)$ & $P(8)-C(32)$ & $186.6(7)$ \\
\hline$O(3)-S(3)$ & $143.6(5)$ & $O(4)-S(4)$ & $143.9(5)$ \\
\hline$S(3)-N(3)$ & $151.9(5)$ & $S(3)-C(41)$ & $174.9(9)$ \\
\hline$S(3)-C(42)$ & $174.6(9)$ & $S(4)-N(4)$ & $154.8(6)$ \\
\hline$S(4)-C(43)$ & $174.8(8)$ & $S(4)-C(44)$ & $177.3(8)$ \\
\hline$C(23)-C(24)$ & $141.1(10)$ & $C(23)-C(28)$ & $144.0(10)$ \\
\hline$C(24)-C(25)$ & $138.4(10)$ & $C(24)-C(29)$ & 151.1(10) \\
\hline$C(25)-C(26)$ & $137.1(10)$ & $C(26)-C(27)$ & $137.5(11)$ \\
\hline$C(26)-C(30)$ & $148.2(10)$ & $C(27)-C(28)$ & $136.8(10)$ \\
\hline$C(28)-C(31)$ & $151.2(9)$ & $C(32)-C(33)$ & $145.2(9)$ \\
\hline$C(32)-C(37)$ & $142.4(11)$ & $C(33)-C(34)$ & $138.1(11)$ \\
\hline$C(33)-C(38)$ & $151.6(11)$ & $C(34)-C(35)$ & $135.5(13)$ \\
\hline$C(35)-C(36)$ & $141.7(11)$ & $C(35)-C(39)$ & $148.1(12)$ \\
\hline$C(36)-C(37)$ & $135.5(11)$ & $C(37)-C(40)$ & $150.9(10)$ \\
\hline
\end{tabular}


Tabelle 3

Ausgewählte Bindungswinkel $\left({ }^{\circ}\right)$

\begin{tabular}{|c|c|c|c|}
\hline$P(3)-P(1)-P(4)$ & $81.6(1)$ & $\mathbf{P}(3)-\mathbf{P}(1)-\mathrm{N}(2)$ & $104.1(2)$ \\
\hline$P(4)-P(1)-N(2)$ & $107.5(2)$ & $P(3)-P(2)-P(4)$ & $82.2(1)$ \\
\hline$P(3)-P(2)-N(1)$ & $101.4(2)$ & $P(4)-P(2)-N(1)$ & $103.4(2)$ \\
\hline $\mathbf{P}(1)-\mathbf{P}(3)-\mathbf{P}(2)$ & 88.7(1) & $\mathbf{P}(1)-\mathbf{P}(3)-\mathbf{C}(1)$ & $113.2(2)$ \\
\hline $\mathbf{P}(2)-\mathbf{P}(3)-\mathrm{C}(1)$ & $104.8(2)$ & $\mathbf{P}(1)-\mathbf{P}(4)-\mathbf{P}(2)$ & $86.7(1)$ \\
\hline$P(1)-P(4)-C(10)$ & $103.2(2)$ & $P(2)-P(4)-C(10)$ & $102.7(2)$ \\
\hline $\mathrm{O}(1)-\mathrm{S}(1)-\mathrm{N}(2)$ & $120.1(3)$ & $O(1)-S(1)-C(19)$ & $109.0(4)$ \\
\hline$N(2)-S(1)-C(19)$ & $104.5(4)$ & $O(1)-S(1)-C(20)$ & $109.0(4)$ \\
\hline$N(2)-S(1)-C(20)$ & $110.5(4)$ & $C(19)-S(1)-C(20)$ & $102.2(4)$ \\
\hline $\mathrm{O}(2)-\mathrm{S}(2)-\mathrm{N}(1)$ & $119.6(3)$ & $O(2)-S(2)-C(21)$ & $109.2(3)$ \\
\hline$N(1)-S(2)-C(21)$ & $108.0(4)$ & $\mathrm{O}(2)-\mathrm{S}(2)-\mathrm{C}(22)$ & $108.9(4)$ \\
\hline$N(1)-S(2)-C(22)$ & $108.0(4)$ & $\mathrm{C}(21)-\mathrm{S}(2)-\mathrm{C}(22)$ & $101.6(4)$ \\
\hline $\mathbf{P}(2)-\mathrm{N}(1)-\mathbf{S}(2)$ & $119.6(3)$ & $P(1)-N(2)-S(1)$ & $129.9(4)$ \\
\hline$P(3)-C(1)-C(2)$ & $130.4(6)$ & $P(3)-C(1)-C(6)$ & $114.5(5)$ \\
\hline$C(2)-C(1)-C(6)$ & $115.0(6)$ & $\mathrm{C}(1)-\mathrm{C}(2)-\mathrm{C}(3)$ & $121.5(7)$ \\
\hline$C(1)-C(2)-C(7)$ & $123.5(7)$ & $C(3)-C(2)-C(7)$ & $115.0(6)$ \\
\hline$C(2)-C(3)-C(4)$ & $121.7(7)$ & $C(3)-C(4)-C(5)$ & $119.6(8)$ \\
\hline$C(3)-C(4)-C(8)$ & $122.1(6)$ & $C(5)-C(4)-C(8)$ & $118.3(7)$ \\
\hline$C(4)-C(5)-C(6)$ & $119.6(7)$ & $C(1)-C(6)-C(5)$ & $122.6(6)$ \\
\hline$C(1)-C(6)-C(9)$ & $123.2(6)$ & $C(5)-C(6)-C(9)$ & $114.2(6)$ \\
\hline$P(4)-C(10)-C(11)$ & $121.5(5)$ & $P(4)-C(10)-C(15)$ & $124.4(5)$ \\
\hline$C(11)-C(10)-C(15)$ & $113.9(6)$ & $C(10)-C(11)-C(12)$ & $120.4(6)$ \\
\hline$C(10)-C(11)-C(16)$ & $124.5(6)$ & $C(12)-C(11)-C(16)$ & $115.1(6)$ \\
\hline$C(11)-C(12)-C(13)$ & $121.7(7)$ & $C(12)-C(13)-C(14)$ & $118.2(7)$ \\
\hline$C(12)-C(13)-C(17)$ & $119.8(7)$ & $C(14)-C(13)-C(17)$ & $122.0(7)$ \\
\hline$C(13)-C(14)-C(15)$ & $121.3(7)$ & $\mathrm{C}(10)-\mathrm{C}(15)-\mathrm{C}(14)$ & $122.5(6)$ \\
\hline$C(10)-C(15)-C(18)$ & $123.6(6)$ & $C(14)-C(15)-C(18)$ & $113.9(6)$ \\
\hline$P(6)-P(5)-P(8)$ & $83.2(1)$ & $P(6)-P(5)-N(3)$ & $106.1(2)$ \\
\hline$P(8)-P(5)-N(3)$ & $103.6(2)$ & $P(5)-P(6)-P(7)$ & $87.8(1)$ \\
\hline$P(5)-P(6)-C(23)$ & $103.6(2)$ & $P(7)-P(6)-C(23)$ & $102.3(2)$ \\
\hline$P(6)-P(7)-P(8)$ & $83.9(1)$ & $\mathbf{P}(6)-\mathbf{P}(7)-\mathrm{N}(4)$ & $103.7(2)$ \\
\hline$P(8)-P(7)-N(4)$ & $102.1(2)$ & $P(5)-P(8)-P(7)$ & $90.7(1)$ \\
\hline$P(5)-P(8)-C(32)$ & $113.2(2)$ & $\mathbf{P}(7)-\mathbf{P}(8)-\mathrm{C}(32)$ & $101.4(2)$ \\
\hline$O(3)-S(3)-(3)$ & $119.5(3)$ & $O(3)-S(3)-C(41)$ & $107.9(4)$ \\
\hline$N(3)-S(3)-C(41)$ & $111.2(4)$ & $O(3)-S(3)-C(42)$ & $109.2(4)$ \\
\hline$N(3)-S(3)-C(42)$ & $104.0(4)$ & $C(41)-S(3)-C(42)$ & $104.0(4)$ \\
\hline$O(4)-S(4)-N(4)$ & $118.9(3)$ & $O(4)-S(4)-C(43)$ & $108.9(4)$ \\
\hline$N(4)-S(4)-C(43)$ & $108.1(4)$ & $O(4)-S(4)-C(44)$ & $109.3(4)$ \\
\hline$N(4)-S(4)-C(44)$ & $108.4(4)$ & $C(43)-S(4)-C(44)$ & $101.9(4)$ \\
\hline$P(5)-N(3)-S(3)$ & $129.4(4)$ & $\mathbf{P}(7)-\mathbf{N}(4)-\mathbf{S}(4)$ & $119.4(3)$ \\
\hline$P(6)-C(23)-C(24)$ & $124.8(5)$ & $P(6)-C(23)-C(28)$ & $120.5(5)$ \\
\hline $\mathrm{C}(24)-\mathrm{C}(23)-\mathrm{C}(28)$ & $114.7(6)$ & $C(23)-C(24)-C(25)$ & $121.6(7)$ \\
\hline$C(23)-C(24)-C(29)$ & $124.4(6)$ & $C(25)-C(24)-C(29)$ & $113.8(6)$ \\
\hline$C(24)-C(25)-C(26)$ & $121.6(7)$ & $C(25)-C(26)-C(27)$ & 118.1(6) \\
\hline$C(25)-C(26)-C(30)$ & $121.5(7)$ & $C(27)-C(26)-C(30)$ & $120.4(6)$ \\
\hline$C(26)-C(27)-C(28)$ & $121.9(7)$ & $C(23)-C(28)-C(27)$ & $121.3(6)$ \\
\hline$C(23)-C(28)-C(31)$ & $124.5(6)$ & $C(27)-C(28)-C(31)$ & $114.0(6)$ \\
\hline$P(8)-C(32)-C(33)$ & $129.3(6)$ & $P(8)-C(32)-C(37)$ & $115.4(5)$ \\
\hline $\mathrm{C}(33)-\mathrm{C}(32)-\mathrm{C}(37)$ & $115.3(6)$ & $C(32)-C(33)-C(34)$ & $121.5(7)$ \\
\hline$C(32)-C(33)-C(38)$ & $123.3(7)$ & $C(34)-C(33)-C(38)$ & $115.2(6)$ \\
\hline$C(33)-C(34)-C(35)$ & $120.3(7)$ & $C(34)-C(35)-C(36)$ & $120.7(8)$ \\
\hline$C(34)-C(35)-C(39)$ & $117.9(7)$ & $C(36)-C(35)-C(39)$ & $121.3(8)$ \\
\hline$C(35)-C(36)-C(37)$ & $119.8(8)$ & $C(32)-C(37)-C(36)$ & $122.4(7)$ \\
\hline$C(32)-C(37)-C(40)$ & $120.9(6)$ & $C(36)-C(37)-C(40)$ & $116.7(7)$ \\
\hline
\end{tabular}


und Analytisches Labor des Instituts für Anorganische Chemie der Universität Göttingen.

$\mathrm{Me}_{2} \mathrm{~S}(\mathrm{O})=\mathrm{NSiMe}_{3}$ [19] und $\left(\mathrm{Ph}_{3} \mathrm{P}\right)_{2} \mathrm{PtC}_{2} \mathrm{H}_{4}$ [20] wurden nach bekannten Verfahren dargestellt.

1. Verbesserte Darstellung von 1,3,5-Tris(trifluormethyl)benzol (I) [7]

In einem 11-Monelzylinder werden $100 \mathrm{~g}(0.47 \mathrm{~mol})$ Benzol-1,3,5-tricarbonsäure vorgelegt und $500 \mathrm{~g}(4.6 \mathrm{~mol}) \mathrm{SF}_{4}$ einkondensiert. Das Reaktionsgemisch wird auf $170^{\circ} \mathrm{C}$ erwärmt und $48 \mathrm{~h}$ bei dieser Temperatur gehalten. Nach Abkühlen auf Raumtemperatur wird das flüssige Reaktionsprodukt auf Eis gegossen, mit Natronlauge $(10 \%, 2 \times 200 \mathrm{ml})$ und Wasser gewaschen und über $\mathrm{CaCl}_{2}$ getrocknet. Destillation under Normaldruck liefert $119.3 \mathrm{~g}(90 \%) 1$ als klare, farblose Flüssigkeit (Kp. 118-120 ${ }^{\circ} \mathrm{C}$ ). Die spektroskopischen Daten stimmen mit den Literaturwerten überein [7].

\section{Dichlor-[2,4,6-tris(trifluormethyl)phenyl]phosphan (3)}

$\mathrm{Zu} 11.4 \mathrm{~g}$ (40.4 mmol) 1 in $25 \mathrm{ml} \mathrm{Et}{ }_{2} \mathrm{O}$ tropft man bei Raumtemperatur $25.2 \mathrm{ml}$ einer n-Butyllithium-Lösung (1.6 $\mathrm{N}$ in n-Hexan) und läßt noch $2 \mathrm{~h}$ nachrühren. Die so erhaltene Lösung von 2 wird bei $-78^{\circ} \mathrm{C}$ zu einem Gemisch von $5.5 \mathrm{~g}$ (40.4 mmol) $\mathrm{PCl}_{3}$ und $25 \mathrm{ml} \mathrm{Et}_{2} \mathrm{O}$ zugetropft. Nach Erwärmen auf Raumtemperatur wird der Ansatz durch eine dünne Schicht Celite filtriert, der Rückstand mit $40 \mathrm{ml} \mathrm{Et}{ }_{2} \mathrm{O}$ gewaschen und das Filtrat nach Abziehen des Lösungsmittels im Vakuum fraktioniert. Man erhält $9.75 \mathrm{~g}(25.4 \mathrm{mmol}, 63 \%) 3$ als farblose, hydrolyseempfindliche Flüssigkeit (Kp. $46-48^{\circ} \mathrm{C} / 0.02$ Torr).

${ }^{1} \mathrm{H}-\mathrm{NMR}$ ( $\mathrm{CDCl}_{3}$, TMS ext.): $\delta 8.29$ (s) ppm. ${ }^{19} \mathrm{~F}\left\{{ }^{1} \mathrm{H}\right\}-\mathrm{NMR}\left(\mathrm{CDCl}_{3}, \mathrm{CFCl}_{3}\right.$ ext.): $\delta-53.7\left(\mathrm{~d}, o-\mathrm{CF}_{3},{ }^{4} J(\mathrm{PF}) 61.4 \mathrm{~Hz}\right),-64.6\left(\mathrm{~s}, p-\mathrm{CF}_{3}\right) \mathrm{ppm} .{ }^{31} \mathrm{P}\left({ }^{1} \mathrm{H}\right\}-\mathrm{NMR}$ $\left(\mathrm{Et}_{2} \mathrm{O} / \mathrm{CDCl}_{3}, 85 \% \mathrm{H}_{3} \mathrm{PO}_{4}\right.$ ext): $\delta 144.7$ (sept, ${ }^{4} J(\mathrm{PF}) 61.4 \mathrm{~Hz}$ ) ppm.

\section{2,4,6-Tris(trifluormethyl)phenylphosphan (4)}

$\mathrm{Zu}$ einer Suspension von $1.2 \mathrm{~g}(31.6 \mathrm{mmol}) \mathrm{LiAlH}_{4}$ in $50 \mathrm{ml} \mathrm{Et}{ }_{2} \mathrm{O}$ tropft man bei Raumtemperatur innerhalb von $30 \mathrm{~min} 19.5 \mathrm{~g}$ (50.9 mmol) 2. Der Ansatz wird $2 \mathrm{~h}$ bei Raumtemperatur gerührt und 1 h unter Rückfluß gekocht. Nach Abkühlen wird durch vorsichtige Zugabe von $2.5 \mathrm{ml}$ Wasser hydrolysiert, der entstandene Niederschlag abfiltriert und mit $2 \times 30 \mathrm{ml} \mathrm{Et}{ }_{2} \mathrm{O}$ gewaschen. Das blaßgelbe Filtrat wird über $\mathrm{CaCl}_{2}$ getrocknet. Destillative Aufarbeitung liefert $9.8 \mathrm{~g}$ (31.2 mmol, 61\%) 4 als farblose, luftempfindliche Flussigkeit, die bei $56-58^{\circ} \mathrm{C} / 12$ Torr siedet. ${ }^{1} \mathrm{H}-\mathrm{NMR}\left(\mathrm{C}_{6} \mathrm{D}_{6}, \mathrm{TMS}\right): \delta 7.74$ (s, CH), 3.93 (d sept, PH, ${ }^{1} J(\mathrm{PH}) 216 \mathrm{~Hz},{ }^{5} J(\mathrm{FH}) 5.2$ Hz) ppm. ${ }^{19} \mathrm{~F}\left\{{ }^{1} \mathrm{H}\right\}-\mathrm{NMR}\left(\mathrm{C}_{6} \mathrm{D}_{6}, \mathrm{CFCl}_{3}\right.$ ext.): $\delta-61.4$ (d, o-CF,$\left.{ }^{4} J(\mathrm{PF}) 29 \mathrm{~Hz}\right)$, -63.6 (d, p-CF,$\left.{ }^{6} J(\mathrm{PF}) 2.3 \mathrm{~Hz}\right) \mathrm{ppm} .{ }^{31} \mathrm{P}\left\{{ }^{1} \mathrm{H}\right\}-\mathrm{NMR}\left(\mathrm{C}_{6} \mathrm{D}_{6}, 85 \% \mathrm{H}_{3} \mathrm{PO}_{4}\right.$ ext.): $\delta$ -139.1 (sept q, ${ }^{4} J(\mathrm{PF}) 29 \mathrm{~Hz},{ }^{6} J(\mathrm{PF}) 2.3 \mathrm{~Hz}$ ) ppm. MS(FI): $m / z 314(M, 100 \%)$.

\section{Bis[2,4,6-tris(trifluormethyl)phenyl]diphosphen (5)}

$6.01 \mathrm{~g}(15.7 \mathrm{mmol}) 3 \mathrm{und} 4.93 \mathrm{~g}(15.7 \mathrm{mmol}) 4$ werden in $150 \mathrm{ml}$ THF gelöst und bei $0^{\circ} \mathrm{C}$ tropfenweise mit $4.8 \mathrm{~g}(31.5 \mathrm{mmol}) \mathrm{DBU}$ in $20 \mathrm{ml}$ THF versetzt. Man läßt auf Raumtemperatur kommen, filtriert und wäscht den Rückstand (DBU $\cdot \mathrm{HCl}$ ) mit $2 \times 50 \mathrm{ml}$ THF. Das gelbe Filtrat wird im Vakuum zur Trockne eingedampft und das Produkt aus heißem Toluol umkristallisiert. Man erhält $6.8 \mathrm{~g}$ (10.9 mmol, 69\%) 5 als blaßgelbe Kristallnadeln vom Fp. $186^{\circ} \mathrm{C}$. 
Gef.: $\mathrm{C}, 35.0 ; \mathrm{H}, 0.8 ; \mathrm{P}, 9.8 . \mathrm{C}_{18} \mathrm{H}_{4} \mathrm{~F}_{18} \mathrm{P}_{2}$ (623.94) ber.: C, 34.6; $\mathrm{H}, 0.7 ; \mathrm{P}, 9.9 \%$.

${ }^{1} \mathrm{H}-\mathrm{NMR}\left(\mathrm{CDCl}_{3}\right.$, TMS ext.): 88.22 (s) ppm. ${ }^{19} \mathrm{~F}\left\{{ }^{1} \mathrm{H}\right\}-\mathrm{NMR}\left(\mathrm{THF} / \mathrm{C}_{6} \mathrm{D}_{6}\right.$, $\mathrm{CFCl}_{3}$ ext.): $\delta-55.6\left(\mathrm{dd}, o-\mathrm{CF}_{3},{ }^{4} J(\mathrm{PF}) 23 \mathrm{~Hz}\right),-63.3\left(\mathrm{~m}, p-\mathrm{CF}_{3}\right) \mathrm{ppm} .{ }^{31} \mathrm{P}\left\{{ }^{1} \mathrm{H}\right\}-$ NMR (THF $/ \mathrm{C}_{6} \mathrm{D}_{6}, 85 \% \mathrm{H}_{3} \mathrm{PO}_{4}$ ext.): $\delta 474\left(\mathrm{~m},{ }^{4} J(\mathrm{PF}) 23 \mathrm{~Hz}\right) \mathrm{ppm}$. MS(FI): $m / z$ $624(M, 100 \%)$.

\section{Dichlor(S,S-dimethylsulfoximino)phosphan (6)}

$\mathrm{Zu} 6.9 \mathrm{~g}(50 \mathrm{mmol}) \mathrm{PCl}_{3}$ in $100 \mathrm{ml} \mathrm{Et}{ }_{2} \mathrm{O}$ tropft man bei $-70^{\circ} \mathrm{C}$ eine Lösung von $5.1 \mathrm{~g} \mathrm{(30.9} \mathrm{mmol)} \mathrm{Me}_{2} \mathrm{~S}(\mathrm{O})=\mathrm{NSiMe}_{3}$ in $20 \mathrm{ml} \mathrm{Et}{ }_{2} \mathrm{O}$. Nach Erwärmen auf Raumtemperatur werden alle flüchtigen Bestandteile im Vakuum abgezogen. Man erhält $5.6 \mathrm{~g}(28.8 \mathrm{mmol}, 93 \%) 6 \mathrm{als}$ farblosen Feststoff vom Fp. $42^{\circ} \mathrm{C} .{ }^{1} \mathrm{H}-\mathrm{NMR}\left(\mathrm{CDCl}_{3}\right.$, TMS ext.): $\delta 3.33\left(\mathrm{~d},{ }^{4} J(\mathrm{PH}) 1.5 \mathrm{~Hz}\right) \mathrm{ppm} .{ }^{31} \mathrm{P}-\mathrm{NMR}\left(\mathrm{Et}_{2} \mathrm{O} / \mathrm{CDCl}_{3}, 85 \% \mathrm{H}_{3} \mathrm{PO}_{4}\right.$ ext.): $\delta 145.4$ (s) ppm.

6. 1,3-Bis(S,S-dimethylsulfoximino)-2,4-bis[2,4,6-tris(trifluormethyl)phenyl]-cyclotetraphosphan (7)

Zu einer Lösung von $4.3 \mathrm{~g}(13.7 \mathrm{mmol}) 4$ und $2.66 \mathrm{~g}(13.7 \mathrm{mmol}) 6$ in $60 \mathrm{ml} \mathrm{THF}$ tropft man bei $-50^{\circ} \mathrm{C} 4.16 \mathrm{~g}(27.4 \mathrm{mmol}) \mathrm{DBU}$, gelöst in $30 \mathrm{ml}$ THF. Beim Auftauen auf Raumtemperatur fällt $\mathrm{DBU} \cdot \mathrm{HCl}$ aus, und die überstehende Lösung färbt sich gelb. Der Niederschlag wird abfiltriert und das Filtrat zur Trockne eingedampft. Durch Extrahieren mit $80 \mathrm{ml}$ Toluol, erneutes Filtrieren und Abkühlen auf $0^{\circ} \mathrm{C}$ ließen sich geringe Mengen 7 (ca. $50 \mathrm{mg}$ ) in Form von gelben Einkristallen isolieren, die direkt für eine Röntgenstrukturanalyse geeignet waren.

7. [ $\eta^{2}$-Dimethylsulfoximino-2,4,6-tris(trifluormethyl)phenyldiphosphen]-bis(triphenylphosphan)platin(0) (8)

$\mathrm{Zu}$ einer Lösung von $1.0 \mathrm{~g}$ ( $3.2 \mathrm{mmol}) 4$ und $0.62 \mathrm{~g}(3.2 \mathrm{mmol}) 6$ in $150 \mathrm{ml} \mathrm{THF}$ tropft man bei $-78^{\circ} \mathrm{C} 0.96 \mathrm{~g}(6.3 \mathrm{mmol}) \mathrm{DBU}$ und läßt noch $30 \mathrm{~min}$ bei $-78^{\circ} \mathrm{C}$ rühren. Man läßt den Ansatz auf $-60^{\circ} \mathrm{C}$ erwärmen und tropft eine Lösung von 2.4 g $(3.2 \mathrm{mmol})\left(\mathrm{Ph}_{3} \mathrm{P}\right)_{2} \mathrm{PtC}_{2} \mathrm{H}_{4}$ in $50 \mathrm{ml}$ THF innerhalb von $30 \mathrm{~min}$ zu. Nach Entfernen der Kühlung wird das Reaktionsgemisch $12 \mathrm{~h}$ bei Raumtemperatur gerührt. Die rotbraune Lösung wird filtriert, das Lösungsmittel im Vakuum abgezogen und der Rückstand aus Toluol/Hexan umkristallisiert. Man erhält $1.6 \mathrm{~g}$ (1.6 mmol, 44\%) 8 als orangefarbenes Pulver, das sich bei $182^{\circ} \mathrm{C}$ zersetzt.

Gef.: C, 48.5; H, 3.3; N, 1.0; P, 10.7. $\mathrm{C}_{47} \mathrm{H}_{38} \mathrm{~F}_{9} \mathrm{NOP}_{4}$ PtS (1154.1) ber.: C, 48.8; $\mathrm{H}, 3.8 ; \mathrm{N}, 1.2 ; \mathrm{P}, 10.7 \%$.

${ }^{19} \mathrm{~F}\left\{{ }^{1} \mathrm{H}\right\}-\mathrm{NMR}\left(\mathrm{C}_{6} \mathrm{D}_{6} / \mathrm{THF}, \mathrm{CFCl}_{3}\right.$ ext.): $\delta-54.8\left(\mathrm{~m}, o-\mathrm{CF}_{3}\right),-64.0\left(\mathrm{~s}, p-\mathrm{CF}_{3}\right)$ ppm. ${ }^{31} \mathrm{P}\left\{{ }^{1} \mathrm{H}\right\}-\mathrm{NMR}\left(500 \mathrm{MHz}\right.$, Toluol $/ \mathrm{C}_{6} \mathrm{D}_{6}, 85 \% \mathrm{H}_{3} \mathrm{PO}_{4}$ ext.): $\delta 130.2$ (m, P-Rf, 1P); 29.5 (m, P-N, 1P); 26.5, 24.5 (m, $\mathrm{PPh}_{3}$, je 1P) ppm.

\section{Fluor-bis[2,4,6-tris(trifluormethyl)phenyl]arsan (9)}

Aus $20.0 \mathrm{~g}$ (70.9 mmol) 1 in $150 \mathrm{ml} \mathrm{Et}{ }_{2} \mathrm{O}$ und $46.1 \mathrm{ml} \mathrm{n}$-Butyllithium-Lösung (1.6 $N$ in Hexan) wird zunächst eine Lösung von 2 hergestellt. Nach $2 \mathrm{~h}$ Rühren bei Raumtemperatur werden $4.67 \mathrm{~g}(35.4 \mathrm{mmol}) \mathrm{AsF}_{3}$ langsam zugetropft. Dabei tritt Erwärmung ein, und ein farbloser Niederschlag fällt aus. Man läßt $12 \mathrm{~h}$ bei Raumtemperatur rühren und erhitzt anschließend noch $3 \mathrm{~h}$ unter Rückfluß. Nach Abziehen des Lösungsmittels im Vakuum wird der Rückstand bei $65^{\circ} \mathrm{C} / 0.01$ Torr sublimiert. Man erhält $15.5 \mathrm{~g}$ (23.6 mmol, 67\%) 9 als farblose Kristalle. ${ }^{1}$ H-NMR 
$\left(\mathrm{C}_{6} \mathrm{D}_{6}\right.$, TMS ext.): $\delta 7.78$ (s) ppm. ${ }^{19} \mathrm{~F}\left\{{ }^{1} \mathrm{H}\right\}-\mathrm{NMR}\left(\mathrm{C}_{6} \mathrm{D}_{6}, \mathrm{CFCl}_{3}\right.$ ext.): $\delta-56.2$ (d, $\left.o-\mathrm{CF}_{3},{ }^{5} J(\mathrm{FF}) 14.7 \mathrm{~Hz}\right),-64.3\left(\mathrm{~d}, p-\mathrm{CF}_{3},{ }^{6} J(\mathrm{FF}) 0.6 \mathrm{~Hz}\right),-189.1\left(\mathrm{~m}, \mathrm{AsF},{ }^{5} J(\mathrm{FF})\right.$ 14.7 Hz) ppm. MS(FI): $m / z 656(M, 30 \%)$.

\section{Bis[2,4,6-tris(trifluormethyl)phenyl]arsan (10)}

$\mathrm{Zu}$ einer Suspension von $0.25 \mathrm{~g}(6.6 \mathrm{mmol}) \mathrm{LiAlH}_{4}$ in $50 \mathrm{ml} \mathrm{Et}{ }_{2} \mathrm{O}$ tropft man 2.8 $\mathrm{g}$ (4.2 mmol) 9, gelöst in $50 \mathrm{ml} \mathrm{Et}{ }_{2} \mathrm{O}$ und erhitzt anschließend $4 \mathrm{~h}$ unter Rückfluß. Nach Abkühlen auf $0^{\circ} \mathrm{C}$ wird mit $3 \mathrm{ml}$ Wasser vorsichtig hydrolysiert und nochmals $4 \mathrm{~h}$ unter Rückfluß gekocht. Der Ansatz wird durch Glaswolle filtriert und das gelbe Filtrat über $\mathrm{CaCl}_{2}$ getrocknet. Sublimation bei $85^{\circ} \mathrm{C} / 0.8$ Torr liefert $2.0 \mathrm{~g}$ (3.1 mmol, 75\%) 10 als farblosen Feststoff.

${ }^{1} \mathrm{H}-\mathrm{NMR}\left(\mathrm{CDCl}_{3}\right.$, TMS ext.): $\delta 8.1(\mathrm{~s}, \mathrm{CH}), 6.3\left(\mathrm{~m}, \mathrm{AsH},{ }^{5} J(\mathrm{FH}) 3.7 \mathrm{~Hz}\right) \mathrm{ppm}$. ${ }^{19} \mathrm{~F}-\mathrm{NMR}\left(\mathrm{CDCl}_{3}, \mathrm{CFCl}_{3}\right.$ ext.): $\delta-58.6\left(\mathrm{~d}, o-\mathrm{CF}_{3},{ }^{5} J(\mathrm{FH}) 3.7 \mathrm{~Hz}\right),-64.1$ (d, $\left.p-\mathrm{CF}_{3},{ }^{7} J(\mathrm{FH}) 0.6 \mathrm{~Hz}\right)$. MS(EI): $m / z 638(M, 10 \%)$.

\section{Röntgenstrukturbestimmung von 7}

$\mathrm{C}_{22} \mathrm{H}_{16} \mathrm{~F}_{18} \mathrm{~N}_{2} \mathrm{O}_{2} \mathrm{P}_{4} \mathrm{~S}_{2}$, monoklin, Raumgruppe $P 2_{1} / n$, Zelldimensionen: $a$ $1569.7(2), b$ 2293.6(3), $c$ 1823.1(2) pm, $\beta$ 94.94(1) ${ }^{\circ}, V=6.5 \mathrm{~nm}^{3}, \rho_{\text {ber. }}=1.77 \mathrm{Mg}$ $\mathrm{m}^{-3}, Z=8, T=-85^{\circ} \mathrm{C}, \mu\left(\mathrm{Mo}-K_{\alpha}\right.$, Graphitmonochromator): $0.48 \mathrm{~mm}^{-1}, 8488$ unabhängige Reflexe, davon 6967 mit $F \geqslant 3 \sigma F, 2 \theta_{\max }=45^{\circ}$, Datensammlung auf einem Stoe-Siemens-AED-Diffraktometer mit Mo- $K_{\alpha}$-Strahlung, Strukturlösung und Verfeinerung mit dem Programmsystem SHELX 86, 906 Parameter wurden verfeinert, alle Nichtwasserstoffatome wurden anisotrop verfeinert. Die Wasserstoffatome wurden geometrisch ideal positioniert und als starre Gruppe nach dem Reitermodell gerechnet. $R=0.074, R_{\mathrm{w}}=0.079, w^{-1}=\sigma(F)^{2} \cdot 0.0008 F^{2}$.

\section{Dank}

Wir danken der Deutschen Forschungsgemeinschaft und dem Fonds der Chemischen Industrie für die großzügige Unterstützung dieser Arbeit.

\section{Literatur}

1 M. Yoshifuji, I. Shima, N. Inamoto, K. Hirotsu und T. Higuchi, J. Am. Chem. Soc., 103 (1981) 4587.

2 A.H. Cowley, Polyhedron, 3 (1984) 389 und dort zitierte Literatur.

3 L. Weber, K. Reizig, D. Bungardt und R. Boese, Organometallics, 6 (1987) 110.

4 J. Escudie, C. Couret, H. Ranaivonjatovo, M. Lazraq und I. Satge, Phosphorus and Sulfur, 31 (1987) 27.

5 M.P. Bigwood, P.J. Corvan und J.J. Zuckerman, J. Am. Chem. Soc., 103 (1981) 7643.

6 G.A. Bosswell, W.C. Ripka, R.M. Schribner und C.W. Tullock, Org. React., 21 (1974) 1.

7 G.E. Carr, R.D. Chambers, T.F. Holmes und D.G. Parker, J. Organomet. Chem., 325 (1987) 13.

8 Euchem Conference, PSIBLOCS, Paris-Palaiseau, 22. August 1988.

9 H.W. Roesky, T. Tojo, M. Ilemann und D. Westhoff, Z. Naturforsch. B, 42 (1987) 877.

10 H.W. Roesky, U. Seseke, M. Noltemeyer, P.G. Jones und G.M. Sheldrick, J. Chem. Soc., Dalton Trans., (1986) 1309.

11 H.W. Roesky, M. Scholz, F. Edelmann, M. Noltemeyer und G.M. Sheldrick, Chem. Ber., 120 (1987) 1882.

12 H.W. Roesky, F. Schrumpf und F. Edelmann, Z. Naturforsch. B, 42 (1987) 874.

13 G. Fritz, T. Vaahs, W. Hönle und H.G. von Schnering, Z. Anorg. Allg. Chem., 552 (1987) 34.

14 G.J. Palenik und J. Donohue, Acta Cryst., 15 (1962) 564.

15 F. Sanz und J.J. Daly, J. Chem. Soc. A, (1971) 1083. 
16 R. Riedel, H.-D. Hansen und E. Fluck, Angew. Chem., 97 (1985) 1056; Angew. Chem. Int. Ed. Engl., 24 (1985) 1056.

17 W. Weigand, A.W. Cordes und P.N. Swepston, Acta Cryst., 37 (1981) 1631.

18 L. Heuer, M. Sell, R. Schmutzler und D. Schomburg, Polyhedron, 6 (1987) 1295.

19 H. Schmidbaur und G. Kammel, Chem. Ber., 102 (1969) 4128.

20 U. Nagel, Chem. Ber., 115 (1982) 1998. 